Francesco Stella

\title{
Théologie de la poésie entre Scolastique et Humanisme
}

\author{
Le statut de la poésie biblique ${ }^{1}$
}

\section{Exclusion de la poésie du système culturel du Moyen Âge}

Je vais partir d'une image (Fig. 1) dont je me sers souvent pour exemplifier l'attitude de la culture médiévale chrétienne envers le statut de la poésie : la miniature qui adorne la feuille 32r du célèbre manuscrit perdu de l'Hortus deliciarum ${ }^{2}$ d'Herrade, abbesse de Hohenbourg au XII ${ }^{\mathrm{e}}$ siècle, qui se trouvait justement ici à Strasbourg.

On y illustre les relations entre les arts et les disciplines dans le système culturel médiéval : au centre du cercle règne Sagesse, soit Philosophia, assise sur un trône sous lequel Socrate et Platon dialoguent, alors que sept niches, disposées en couronne autour du cercle, encadrent les sept arts libéraux. En dehors du cercle, en bas, apparaissent quatre figures de scribes, ou écrivains, caractérisées par un inspirateur négatif : un petit oiseau noir, représentant le démon, qui suggère dans leurs oreilles le contenu des écritures in fieri. La légende transversale nous explique qu'il s'agit des poetae, vel magi, spiritu immundo instincti («les poètes, ou magiciens, inspirés par un esprit immonde»). Et, entre l'un et l'autre de leurs sièges, des lettres spécifient : Isti immundis spiritibus, scribunt artem magicam et poetriam id est fabulosa commenta («ceux-ci, inspirés par les esprits immondes, écrivent des traités d'arts magiques et poétiques, c'est-à-dire des inventions fantastiques»). Les poètes sont donc des exécuteurs du malin, identifiés aux magiciens en tant que producteurs d'une réalité étrangère, d'une fiction de l'esprit humain, et, en qualité de magiciens, ils sont exclus du système culturel et bannis de ses marges ${ }^{3}$.

Une condamnation et des effets pareils sur le système des genres littéraires sont repérables dans la culture islamique, et arabe et persane, comme je l'ai écrit ailleurs (Stella 2003).

1 J'utilize ici des parties de mon essai italien Stella (2010) 251-328 et 350-357.

2 Chef-d'oeuvre d'Herrade de Landsberg, abbesse de Hohenbourg en Alsace au XII siècle. Texte et images dans l'édition de Gillen (1979) ; les symboles sont commentés dans Cames (1971) ; Nonn (2012); Mersch (2012); Joyner (2016).

3 Cfr. Gompe (1973) ; Verdier (1969) ; Pagani (1992) 151.

https://doi.org/10.1515/9783110687224-031 


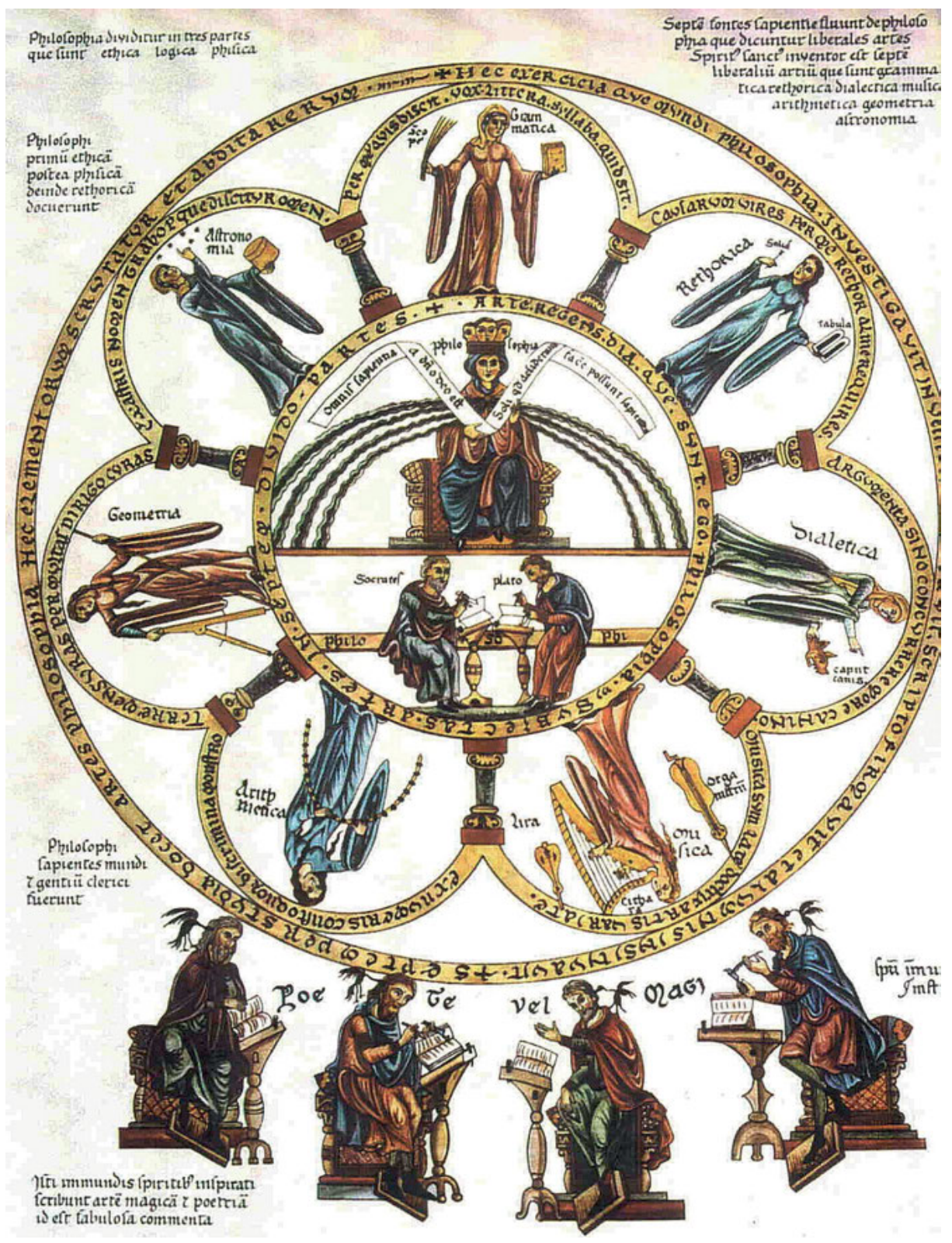

Fig. 1: $f$. 32r Hortus deliciarum d'Herrade de Landsberg (manuscrit perdu) 


\section{Les Summae scolastiques}

\section{Le Decretum Gratiani}

Juste avant que l'Hortus deliciarum soit composé (1140), le Decretum Gratiani ${ }^{4}$, qui recueillait et harmonisait la législation canoniste de l'Église dans un ouvrage qui a servi de texte de référence pendant des siècles, paraît codifier aux clercs l'interdiction de cultiver la poésie ${ }^{5}$. Il la tire d'Isidore ${ }^{6}$, où le titre est explicitement Quare prohibetur Christianus poetica figmenta legere, qui adresse la prohibition à tous les fidèles, alors que la Distinctio est entièrement destinée à la formation cléricale. Quelle en est la raison? "C'est que, à travers leurs attraits, les histoires excitent excessivement l'esprit à la stimulation des passions ; car non seulement elles célèbrent les démons, les encensant, mais elles reportent aussi volontiers leurs dictons ». Pourtant Gratien, dans sa réponse (pars V), réplique que, pour le prêtre, le pire des malheurs est l'ignorance, puisqu'elle remet la conduite d'une âme dans les mains d'autrui ; l'ignorance est donc à éviter autant que la peste. La conséquence non explicite est que finalement le danger dérivant d'un renoncement à la lecture de la poésie (terme qui désigne inévitablement la poésie classique) est plus grand que celui auquel expose la lecture de ses parties les plus lascives.

\section{Alexandre d'Halès (Hales, Hayles)}

$\mathrm{Au}$ XIII ${ }^{\mathrm{e}}$ siècle, le thème de la poésie devient l'objet de commentaires rapides dans les grandes synthèses théologiques des intellectuels appartenant aux ordres mendiants, destinées aux écoles de théologie des universités. Comme Curtius ${ }^{7}$ le rappelle déjà, la summa circulant sous le nom du franciscain anglais (de formation et enseignement parisiens) Alexandre d'Halès (Hales, 1183-1245) ${ }^{8}$ s'ouvre précisément sur la question «Si la théologie est une science», et, à l'article I du IV chapitre, se demande «an modus sacrae Scripturae sit artificialis vel scientialis ${ }^{9}$, c'est-à-dire "si le genre des Saintes Écritures est technique [rhétorique] ou scientifique», ce qui introduit ou confirme une distinction polaire entre des approches gnoséologiques

4 Avec ce titre est connue la Concordantia discordantium canonum rédigée par le moine Gratien. 5 Chap. 15 de la distinctio 37, fondé sur Isidore In libro Sententiarum III de summo bono 13. Cf. Meersseman (1958).

6 Sententiae, PL 83, 685 A; reprise par Ive de Chartres Decretum P.L. 161, 303C et Abélard Theologica christiana, PL 178, 1208 D.

7 Curtius (1992) 239-253 : sur les summae voir 248-250.

8 L'ouvrage a été écrit par lui et d'autres confrères (Jean de la Rochelle, Oddon de Rigaud et Guillaume Meliton).

9 Summa theologiae I.1, Quaracchi (1924) 7. 
habituellement superposées ou confondues ${ }^{10}$. L'objection (nécessairement présente dans le genre de la quaestio) déclare que le mode d'exposition de la Bible ne répond pas à des critères scientifiques ni à des critères techniques, car en fait theologicus modus est poeticus ${ }^{11}$. La responsio d'Alexandre, basée sur Augustin ${ }^{12}$, surmonte le problème en expliquant que les Écritures sont au-delà des séparations ars/science ou poésie/ars/science, et que, lorsqu'elles emploient des techniques poétiques, c'est à la fois pour suppléer l'insuffisance de la capacité humaine de compréhension et pour voiler leur sens à ceux qui ne sont pas dignes de le saisir. Pour soutenir sa thèse, il cite un passage de la Hiérarchie céleste ${ }^{13}$ de l' "heureux Denys», soit Denys pseudo-Aréopagite, où celui-ci avance que «la théologie s'est servie avec adresse de figures sacrées pour la compréhension de réalités non figurables» et qu'«il n'est pas possible que le rayon divin nous éclaire sans être voilé par la variété des couvertures anagogiques» : c'est pourquoi les Saintes Écritures font recours à de «belles représentations figurées de ce qui n’a pas de figure» («pulchre in sacra Scriptura procreatae sunt informium formae et figurae carentium figuris»). Les raisons qui justifient cet usage relèvent, d'un côté, de la nécessité de l'intellect humain, qui n'arrive pas à la pleine compréhension de la réalité divine, et, de l'autre, de l'opportunité de cacher aux méchants la dignité du Vrai. La Bible se distingue donc nettement de la poésie, mais aussi de la philosophie (mieux : des méthodes dialectiques de ses traités scientifiques) ; voilà pourquoi, même si parfois ses techniques d'expression coïncident avec celles d'une science ou d'un art, elles ne sont jamais assimilables à celles-ci.

\section{L’argument du Pseudo-Denys}

Denys, cité dans la Summa d'Alexandre, partageait le présupposé ${ }^{14}$ néo-platonicien sur l'impossibilité d'exprimer Dieu par toute forme d'expression du langage humain $^{15}$. La conséquence, au niveau pratique, est la nécessité de parler de Dieu par

10 Vincent de Beauvais, par exemple, soutenait que art, science et doctrine sont synonymes: Speculum doctrinale I.13: Idem est ars et scientia, doctrina quoque, disciplina et facultas. De façon analogue ils s'étaient exprimés Aristote Metaph. I.1 e II.468, Isidore Etym. I.1 (PL 82, 73) et Hugues de Saint-Victor Eruditio didascalica II.1 (PL 176, 751).

11 Omnis modus poeticus est inartificialis sive non scientialis, quia est modus historicus vel transumptus, qui quidem non competunt arti, sed theologicus modus est poeticus vel historialis vel parabolicus; ergo non est artificialis. Sur le rôle de la poésie dans l'éducation franciscaine cf. Roest (2004). 12 Trin. XIV.1, n. 3 (PL 42, 1037).

13 Comme on le sait, il s'agit d'une partie de la Hiérarchie céleste de Denis.

14 Il est connu en Occident par les traductions d'Ilduin, abbé de Saint-Denis à l'époque carolingienne (827), de Jean Scot Erigène (860 - 862), les plus important philosophe du haut moyen âge, et après de Jean Saracène (XII ${ }^{\mathrm{e}}$ s., la version connue par Thomas d'Aquin).

15 Pour ne donner qu'un example de cette théorie, on peut citer De praedestinatione de Jean Scot, dans lequel on affirme explicitement que: nihil digne de deo dicitur, omnia paene sive nominum sive 
images et par métaphores ${ }^{16}$, ainsi que le théorise, en latin, Jean Scot, au IX ${ }^{\mathrm{e}}$ siècle, justement dans son commentaire de Denys. Donc, Denys-Érigène établissent une analogie entre les procédés des Saintes Écritures et ceux de la composition poétique, en partant de leur nécessité partagée d'une narrativité figurée. Ainsi définissent-ils le pivot central de toutes les légitimations ultérieures de la vérité poétique comme instrument de connaissance non indigne de la culture chrétienne :

«De même que l'art poétique présente une doctrine morale ou naturelle à travers des histoires inventées et des similitudes allégoriques, dans le but de faire travailler l'esprit humain (en effet, ceci caractérise les poètes épiques, qui célèbrent les exploits et les conduites des héros à travers des symboles), ainsi la théologie, telle une poétesse [ou bien : telle une composition poétique ${ }^{17}$, adapte les Saintes Écritures à l'utilité de notre esprit, afin de le reconduire, par des fantaisies fictionnelles, à la connaissance parfaite des réalités intelligibles, comme d'un état d'enfance imparfaite jusqu'à une sorte de maturité intérieure».

Cette affirmation n'entraîne pas pour autant une justification, et moins encore un ennoblissement de l'activité poétique en tant que telle. Il est vrai que, dans ce même commentaire, l'Érigène développe un parcours de légitimation des arts basé sur l'interprétation du réel comme théophanie, où les œuvres d'art deviennent elles aussi manuductiones ad Deum, toutefois, parmi ces arts, il ne cite pas la poésie, et il ne rappelle que la musique et; l'art plastique.

De la même manière, lorsque, au XII ${ }^{\mathrm{e}}$ siècle, Anselme de Laon et Abélard développeront l'analyse rhétorique et stylistique de la Bible, dans la lignée des prémisses de Bède et des Carolingiens ${ }^{18}$, l'apologie de l'esthétique biblique ne produit pas le passage à une acceptation de la poésie en tant que art. Au contraire, dans sa Theologia Christiana, Abélard, bien qu'il soit l'auteur raffiné d'hymnes et de planctus bibliques, répète à ce sujet les anathèmes platoniques d'Isidore et Gratien contre les

verborum aliarumque orationis partium signa proprie de Deo dici non posse. Quomodo enim signa sensibilia, id est corporibus adhaerentia remotam illam omni corporeo naturam ad liquidum significare possent, quae vix purgatissima mente attingitur, omnem transcendens intellectum? Eis tamen utitur humane ratiocinationibus post peccatum primi hominis laboriosa egestas, ut quodammodo credatur et innuatur copiosa conditoris sublimitas (Cap. IX, PL 122, 390B).

16 Translative de Deo praedicari: chap. X, col. 394 A.

17 Super Ier. Cael. Sancti Dionysii, PL 122, col. 146 B-C: Quemadmodum ars poetica per fictas fabulas allegoricasque similitudines moralem doctrinam physicam componit ad humanorum animorum exercitationem (hoc enim proprium est heroicorum poetarum, qui virorum fortium facta et mores figurate laudant): ita theologia veluti quaedam poetria sanctam Scripturam fictis imaginationibus ad consultum nostri animi et reductionem a corporalibus sensibus exterioribus, veluti ex quadam imperfecta pueritia, in rerum intellegibilium perfectam cognitionem, tamquam in quadam interioris hominis grandaevitatem, conformatseu. «Poème» est la traduction de poetria choisie par De Bruyne (1998) I. 344. Sur ce passage voir Dronke (1977).

18 Lobrichon (1985) 167. Voir aussi Colnago (2009). 
poètes mensongers ${ }^{19}$. Seuls les adeptes de l'école de Chartres $^{20}$, à travers la théorie de l'integumentum ou involucrum, reconnaîtront avec Bernard la fonction introductoria des poètes par rapport à la philosophie, et accepteront, avec Alain de Lille et Guillaume de Conches, la nécessité d'un discours symbolique sur Dieu ${ }^{21}$, revendiquant en même temps une capacité de communication propre à la poésie ${ }^{22}$.

Bientôt, pourtant, cet argument analogique - avant qu'il ne soit vigoureusement repris par l'humanisme - s'affaiblit, même indépendamment de la réaction scolastique : devant la floraison de la poésie des troubadours, qui envahit les cours avec son prestige nouveau, Jean de Salisbury, bien qu'il se soit probablement formé à l'école de Chartres et qu'il défende, dans le Metalogicon (1159) ${ }^{23}$, l'utilité didactique de la Poésie, déqualifie l'ennoblissement qu'on revendique pour celle-ci en raison de ses affinités avec la polysémie biblique : selon lui, seules les Saintes Écritures peuvent soutenir le véritable sens allégorique. Les auteurs humains, soit qu'ils écrivent au sens propre qu'au sens figuré, doivent rester compréhensibles en soi. Il y a ici une consonance fondamentale, ante litteram, avec l'argument thomiste qui distinguera le langage figuré de l'allégorie in factis, laquelle n'est présente que dans la Bible et est inépuisable, du sensus parabolicus, lequel, au contraire, peut caractériser l'écriture humaine aussi, quoique toujours sous l'aspect du sensus litteralis, c'est-à-dire de l'intention de l'écrivain.

\section{Albert le Grand}

Dans la Summa theologiae, l'éminent dominicain Albert le Grand ${ }^{24}$ (1206-1280) reprend strictement l'argumentation d'Alexandre d'Halès ${ }^{25}$ et s'interroge de modo theologiae en questionnant justement l'idée que, étant donné la nature mystique et «cachée» de la communication (traditio) biblique, les Saintes Écritures n’emploient

19 Theologia Christiana, II livre, conclusion, PL 178, 1207 C (et alibi). Unde et a sanctis postmodum Patribus non incongrue liberalium artium studia, tanquam sacrae paginae admodum necessaria, plurimum commendantur, cum omnino poetica figmenta Christianis interdicantur, non solum quia falsitate referta sunt, et os quod mentitur occidit animam (Sap. I, 11), verum etiam, quia inanium fabularum cogitationibus ad desideria turpitudinum quae fingunt, alliciunt animum, atque a sacrae lectionis meditatione nos abducunt.

20 Les exemples cités par Chevalier (2000) sur la base de Pépin (1970) sont les Allegories d'Homer d'Eraclite, L'antre des nymphes de Porphyre et la Théologie platonique de Proclus.

21 Alain de Lille, Theologicae Regulae et Quoniam homines (ed. Glorieux 1953).

22 En poésie, à la suavitas du sens littéral et à l'instructio moralis se joint l'acutior allegorie subtilitas: Pagani (1995) spec. 155.

2342.32 ss.: il se limite toutefois à en confirmer la position subordonnée à la grammaire, pour en éviter l'autonomie et la conséquente exclusion du système des artes.

24 Poeticus modus infirmior est inter modos philosophiae (Curtius (1992) 249).

25 Dans la cinquième quaestio du Ie livre: I q. membrum 1, ed. Lyon 1651, vol. XVII p. 13², cité par Curtius (1992) 249. 
pas de mode scientifique ni artificiel car elles veulent s'adapter à la compréhension de tout le monde, et non seulement des compétents. Et, pour expliquer comment cela peut arriver, il recourt à l'exemple de la chauve-souris, qui ne perçoit la lumière que si elle est immergée dans le noir, en s'appuyant sur toute une série d'autorités (de Denys jusqu’à Augustin, Grégoire, Boèce) reprises d'Alexandre. En particulier, citant lui aussi la Hiérarchie Céleste, il en conclut que «traitant d'une lumière imperceptible, la théologie emploie au sens propre des expressions figuratives quasi poeticis, à travers ce que Denys ${ }^{26}$ définit materialis manuductio, soit guide matériel. Les autres sciences philosophiques se trompent lorsqu'elles emploient ce » code «, car elles obscurcissent leur énoncé.

\section{Thomas d'Aquin}

Pour Thomas d'Aquin ${ }^{27}$ aussi - dont on a pu explorer tous les passages de textes portant sur la poétique grâce à l'Index du père Busa - la poésie n'est pas en elle un objet théologique, mais plutôt un thème occasionnel, que la théologie rencontre lorsque l'on parle du statut des arts humains ou des critères de la communication sacrée. Mais l'argumentation, ainsi que le genre d'autorités qu'il invoque, sont sensiblement différents de celles des maîtres qui l'avaient précédé, et révèlent une organisation rationnelle, propre à lui, qui reprend des textes d'Augustin autres par rapport à ceux que l'on évoquait d'habitude dans ce débat, en réintroduisant des sujets oubliés, tel celui des poètes théologiens, selon l'organisation du discours établi dans les interrogations anciennes, aristotéliques et patristiques, et en reléguant la présence de Denys et de la théologie apophatique à une position marginale. Ainsi offre-t-il une analyse fine et aiguë du mécanisme sémiotique.

Nous ne nous arrêtons que sur deux morceaux, schématisant une situation $d u$ texte bien autrement complexe. Le premier se trouve dans le Commentaire aux Sentences de Pierre Lombard. Thomas le composa pendant la première période de son magistère à Paris, entre 1252 et 1257. Dans la quaestio unica du premier livre sur le rapport entre les arts et la théologie $\mathrm{e}^{28}$, le troisième argument soutient qu'il ne doit pas y avoir une seule méthode pour des sciences différentes. Notamment la poétique, quae minimum continet veritatis, maxime differt ab ista scientia, quae est verissima. Ergo, cum illa procedat per metaphoricas locutiones, modus huius scientiae non debet

26 Chap. 1.3 ( $P G$ 3, $121 \mathrm{~cd})$.

27 Curtius curieusement ne l'inclut pas dans son aperçu.

28 L'article cinquième en particulier pose le problème du critère (modus procedendi), en se demandant s'il soit artificialis, c'est-à-dire s'il corresponde aux procedure correctes de l'art (dialectique): si - comme on le dirait aujourd'huy - il soit scientifique. Ainsi l'intend aussi le commentaire à la version française de l'oeuvre, p. 40, en ajoutant que che «lorsque Thomas reprendra le sujet dans la Summa theologiae, il mettra la question dans ces termes: cette doctrine est soumise à l'argumentation. Ici il trait le même sujet en considerant surtout la méthode». 
esse talis : «[la poétique] qui contient le moins de vérité, est la plus éloignée de cette science $^{29}$, qui est la plus vraie. Donc, étant donné qu'elle procède par des expressions métaphoriques, le mode de cette science ne saurait être le même ${ }^{30}$.

C'est donc une double question qui émerge ici : d'un côté, la théorie sur le statut de la poésie, ici définie, en passant, comme discipline contenant le moins de vérité, selon les positions traditionnelles. De l'autre, la conclusion selon laquelle le mode expressif qui est propre à la poésie ne saurait être adopté dans l'argumentation théologique, ce qui paraît nier frontalement la tradition de Denys.

Selon Umberto Eco, l'assertion sur l'infima doctrina «ne doit pas être entendue comme un rabaissement de la poésie, ni comme une définition de l'acte poétique tenu, pour user de la terminologie du XVIII ${ }^{\mathrm{e}}$ siècle, pour une perceptio confusa. Il s'agit plutôt de reconnaître à la poésie son titre à figurer au rang des arts (et, donc, sa qualité de recta ratio factibilium), étant admis d'autre part que cette opération, ce facere, ce faire demeure inférieur par nature à la connaissance épurée que procurent la philosophie et la théologie. [...] En fait, à l'instar de tous les penseurs scolastiques, il n'éprouve aucun intérêt pour une théorie de la poésie : un sujet bon pour les spécialistes de rhétorique, lesquels professeraient à la faculté des Arts, et non à la faculté de Théologie» ${ }^{31}$. En fait, un peu plus loin, dans l'argument V, on réaffirme que chaque science a sa méthode, et, dans le $\mathrm{III}^{\mathrm{e}}$, on lit que ${ }^{32}$ «la poétique est la science de ce que la raison ne peut saisir par manque de vérité : il est donc inévitable que la raison soit conduite comme par similitudes ; le mode symbolique est donc commun à l'une et à l'autre, étant donné que ni l'une ni l'autre ne sont adéquates la raison». Cet argument, quoiqu'il soit avancé par nécessité du débat, paraît limiter l'enjeu du précédent, et ouvrir une porte à la justification de l'emploi du langage métaphorique, qui vise à suppléer un défaut de vérité - et donc de capacité d'expression rationnelle - des objets des deux sciences : les fictions poétiques et Dieu, justification sur laquelle se basera la défense humaniste de la poésie.

29 Trop faible la traduction française: «est très différente de cette science». Dans Ia q. 1 a. 8 on réaffirme que «procéder par similitudes diverses et rappresentations sensibles c'est propre de la poétique ».

30 Claudio Mésoniat observe que dans le système thomiste de classement des sciences la poétique est comprise entre les sciences pratiques, qui incluent les arts mécaniques, inférieures en dignité aux spéculatives, tandis qu'Aristote avait concédé à la poétique «uno statuto singolare, non riducibile a quello delle scienze speculative né a quello delle scienze pratiche». Dans l'oeuvre de Thomas un classement semblable de la poétique ne paraît pas exprimé de façon explicite, mais on le déduit de ce qu'on en dit dans les passages mentionnés plus haut: Mésoniat (1984) 16 n. 18. Autre bibliographie citée par lui Mariétan (1901) 177-179 et 192 ; Eco (1975) 1-7 et 202-204.

31 Eco (1987), 94-95, trad. fr. 1997127.

32 Poetica scientia est de his quae propter defectum veritatis non possunt a ratione capi: unde oportet quod quasi quibusdam similitudinibus ratio seducatur: tehologia autem est de his quae sunt supra rationem; et ideo modus symbolicus utrique communis est, cum neutra rationi proportionetur. 
Le texte ${ }^{33}$ de Thomas qui aura influé plus que d'autres sur le débat pré-humaniste $^{34}$, se trouve dans le commentaire à la Métaphysique, où, à propos des philosophes présocratiques, il signale que, chez les Grecs, certains poetae theologi furent célèbres, qui étaient appelés ainsi car de divinis carmina faciebant (ils composaient des poèmes sur les réalités divines «), et il en rappelle trois, Orphée, Musée et Linos, contemporains des Juges du peuple juif ${ }^{35}$. Ces poètes étudièrent quelques aspects de la nature à travers quibusdam aenigmatibus fabularum, soit travers des histoires allégoriques. ${ }^{36}$ Dans Metaphisica 1000a9, Aristote cite Hésiode (auteur d'une cosmogonie) et » d'autres théologiens «, tandis que, dans d'autres passages ${ }^{37}$, il identifie les théologiens aux spécialistes de la nature, et les distingue des philosophes en raison de la nature scientifique de leur pensée. Pourtant, il ne cite pas les trois poètes mentionnés par Thomas (Orphée, Musée et Linos), qui les tire d'une autre source. Le texte d'Aristote, déjà cité par Lactance dans le $D e$ ira $D i^{38}$, qui était pourtant peu lu au Moyen-Âge, avait été employé par Augustin dans plusieurs morceaux de La Cité de

33 On renonce ici pour manque d'éspace à analyizer d'autres passages pertinents. Dans I.l.1 n. 6 il écrit poetae est inducere ad aliquod virtuosum per aliquam decentem repraesentationem, c'est-à-dire que «propre du poète c'est de conduire vers un objectif virtueux par une réprésentation esthétique», en n'excluant pas donc un rôle positif de la poésie au niveau pratique, tout en ayant nié un statut théorique.

34 La source aristotelique ici commentée sera la base soit de la critique du dominicain Giovannino da Mantova, soit des justifications humanistiques de la poésie.

3515 Ad cuius evidentiam sciendum est, quod apud Graecos primi famosi in scientia fuerunt quidam poetae theologi, sic dicti, quia de divinis carmina faciebant. Fuerunt autem tres, Orpheus, Museus et Linus, quorum Orpheus famosior fuit. Fuerunt autem tempore, quo iudices erant in populo Iudaeorum. Unde patet, quod diu fuerunt ante Thaletem, et multo magis ante Aristotelem qui fuit tempore Alexandri. Isti autem poetae quibusdam aenigmatibus fabularum aliquid de rerum natura tractaverunt. Dixerunt enim quod Oceanus, ubi est maxima aquarum aggregatio, et Thetis, quae dicitur dea aquarum, sunt parentes generationis: ex hoc sub fabulari similitudine dantes intelligere aquam esse generationis principium. Cfr. Super Meteora II.1 n. 3. [...] Circa primum duo facit: primo ponit opiniones antiquorum theologorum; secundo naturalium, ibi: qui autem sapientiores etc. Circa primum sciendum est quod ante tempora philosophorum, fuerunt quidam qui vocabantur poetae theologi, sicut Orpheus, Hesiodus et Homerus: quia sub tegumento quarundam fabularum, divina hominibus tradiderunt. De his ergo dicit quod posuerunt quod mare habeat fontes proprios ex quibus causatur. [... ]. Sententia Metaphysicae lib. III.11 n. 3.[...]. Tertio se excusat a diligentiori improbatione huius positionis, ibi, sed de fabulose etc. Circa primum considerandum est, quod apud Graecos, aut naturales philosophos, fuerunt quidam sapientiae studentes, qui deis se intromiserunt occultantes veritatem divinorum sub quodam tegmine fabularum, sicut Orpheus, Hesiodus et quidam alii: sicut etiam Plato occultavit veritatem philosophiae sub mathematicis, ut dicit Simplicius in commento praedicamentorum. Dicit ergo, quod sectatores Hesiodi, et omnes, qui dicebantur theologi, curaverunt persuadere solis sibi, et nos alios spreverunt; quia scilicet veritatem, quam intellexerunt, taliter tradiderunt, quod eis solum possit esse nota. [...]. 36 Parmi eux il y les premiers qui, «à l'époque archaïque, firent des dieux l'objet d'une réflexion» (hoi prótoi ... theologhésantes) en enseignant qu'Océan et Téthys avaient engendré le monde et que les dieux erraient dans le Styx: Arist. Metaph. 939b 29.

37 Curtius (1992) 243.

38 11.8. 
$D_{i e u^{39}}$ : en particulier, au chapitre $14 \mathrm{du}$ livre $\mathrm{XVIII}^{40}$, il rappelle que, à l'époque historique où vécurent les Juges du peuple hébraïque, existaient les poetae, qui etiam theologi dicerentur, quoniam de diis carmina faciebant. Mais il spécifie tout de suite que ces dieux étaient de grands hommes ou des éléments de la nature, et que, si parmi tant de faussetés ou vanités, ces poètes chantèrent quelque chose du Dieu unique et vrai : en le confondant parmi d'autres divinités fausses, ils ne lui furent pas avantageux, Orphée, Musée et Linos non plus, car eux-mêmes ne purent éviter l'indécence des fables mythologiques. La sévère condamnation est donc répétée et précisée dans ce passage, qui, de toute évidence, est la source originale, quoique non déclarée, de Thomas $^{41}$.

Les attestations relatives au rapport de la poésie et de la théologie à la Bible, dans les summae scolastiques, paraissent parfois exclure, parfois soutenir une continuité technique, et donc une possibilité de comparaison entre les deux "genres», sans pourtant exprimer une véritable condamnation de la poésie pareille à celles que lui réserve la théologie patristique et dialectique inspirée par Platon. Comme Curtius l'avait bien compris ${ }^{42}$, Thomas ne discute pas les raisons pour lesquelles la poétique est le dernier des arts, puisqu'il «[...] n’a aucune raison pour la discuter ou la réexaminer. La Scolastique ne s'intéresse pas d'ennoblir la poésie ; elle n'a produit ni

39 Dans le livre VI, dedié à la théologie payenne, le chapitre 5 trait des trois types de théologie exposés par Varron (mythique, naturelle et civile, où d'après Varron mythicon appellant quo maxime utuntur poetae) et le 6 de la théologie mythica, id est fabulosa, qu'Augustin critique en tant que matière de poétes, théâtre, spectacle, où est réprésentée la vie de divinités immorales. La théologie fabulosa est celle qui de diis nefanda figmenta hominum carminibus personat, c'est-à-dire 'fait resonner dans les poèmes abominables des inventions humaines sur les dieux'.

40 Per idem temporis intervallum exstiterunt poetae, qui etiam theologi dicerentur, quoniam de diis carmina faciebant, sed talibus diis, qui licet magni homines, tamen homines fuerunt aut mundi huius, quem verus Deus fecit, elementa sunt aut in principatibus et potestatibus pro voluntate Creatoris et suis meritis ordinati, et si quid de uno vero Deo inter multa vana et falsa cecinerint, colendo cum illo alios, qui dii non sunt, eisque exhibendo famulatum, qui uni tantum debetur Deo, non ei utique rite servierunt nec a fabuloso deorum suorum dedecore etiam ipsi se abstinere potuerunt Orpheus, Musaeus, Linus. Verum isti theologi deos coluerunt, non pro diis culti sunt; quamvis Orpheum nescio quomodo infernis sacris vel potius sacrilegiis praeficere soleat civitas impiorum.

41 L'unique autre auteur médiéval qui mentionne cette sequence est Philippe d'Harveng, perçant exégète et théorique de l'exégèse, dans De institutione clericorum III.45, PL 203 1019a: Et quia studium eorum fuit verbosa de diis carmina fabricari, theologi meruerunt generaliter nominari, gratum habentes tale sibi vocabulum assignari, in quo posset eorum quasi divinum officium designari. Quorum priores et antiquiores Orpheus, Linus, Musaeus fuisse referuntur, qui suis contemporaneis fama et scientia praeferuntur, quorum labore litterario, diis falsis laudes et carmina deferuntur, et hominibus erroneis documenta placita conferuntur. (1020 A) Eo autem tempore quo poetae in Graecia claruerunt et famae testimonio litteralis artem scientiae tenuerunt, in terra promissionis iam populus Israel versabatur, ibique a iudicibus, et, ut quidam aestimant a Debbora iudice regebatur.

42 Curtius (1992) 199 ; 249. Une discussion intéressante sur le placement des livres de poésie dans les bibliothèques scolaires est celle proposée par Franklin-Brown (2015). 
une poétique ni une théorie esthétique ${ }^{43}$. [...] La Scolastique, produite par la dialectique du XII ${ }^{\mathrm{e}}$ siècle, ne conserve pas l'opposition aux auctores, à la rhétorique et à la poésie ; elle ôte de l'aristotélisme la justification philosophique de la poésie». Pourtant, c'est bien l'argument augustinien et thomiste des poetae theologi qui soutiendra la légitimation de la poésie classique.

\section{Le passage de la Scolastique à la discussion humaniste}

Comme on le sait, la qualité du débat change radicalement à partir d'Albertino Mussato (1261-1329), notaire et dramaturge de Padoue, qui reprend les suggestions pseudo-denysiennes, élaborées par Jean Scot et par l'école de Chartres ${ }^{44}$, sur l'équivalence entre poésie et théologie, visant à attribuer à la poésie une position de primauté dans le système de la culture. Selon la lecture vulgarisée d'abord par Garin et Ronconi, puis par d'autres, cette approche aurait été contrecarrée par les penseurs thomistes, tandis qu'elle aurait été adoptée par l'Épître à Cangrande della Scala que l'on attribue à Dante, et, ultérieurement, par Pétrarque ${ }^{45}$, et Boccace $^{46}$; cela ouvre la voie à la sacralisation de la poésie qui, à partir de Coluccio Salutati ${ }^{47}$, mènera jusqu'à Scaliger ${ }^{48}$ et au-delà.

Mais, au cours des dernières années, Thomas Ricklin a accompli des progrès importants, bien que méconnus, dans le repérage de traités de logique modistes qui sont à la source de son approche, alors que Casadei et d'autres ont puissamment remis en cause la paternité de l'Épître. Les positions de ceux qui attribuent à Dante l'anticipation de la théorie du "poète théologien», en sortent donc affaiblies. En fait - comme j'ai déjà essayé de le démontrer ailleurs ${ }^{49}$-, que l'auteur soit ou non Dante, il reste que celui-ci applique à la Comédie, tout comme à l'un de ses chants du

43 C'est important de rappeler ici la note de bas de age de Curtius (1992) n. 23, sans la quelle non ne comprend pas sa position, qui s'oppose aux efforts (à nos jours, les exemples les plus célébres sont Eco (1956) et Biffi (2005)) d'individuer une esthétique dans la doctrine de la Scholastique: «Quando, nella filosofia scolastica, si parla di bellezza, si intende con ciò un attributo di Dio. La metafisica della bellezza (ad es. in Plotino) e la teoria dell'arte non hanno assolutamente nulla in comune. L'uomo »moderno« sopravvaluta smisuratamente l'arte perché ha perduto il senso della bellezza intelligibile, che invece possedevano il neoplatonismo e il Medioevo. Agostino (Conf. X.27.38) si rivolge a Dio: Sero te amavi, pulchritudo tam antiqua et tam nova, sero te amavi [Tardi giunsi ad amarti, o bellezza così antica e così nuova; tardi giunsi ad amarti]. È evidente che qui si tratta di una bellezza del tutto estranea all'estetica.»

44 Sur ça voir la syntèse de Lemoine (1998).

45 Fam. X.4.1: parum abest quin dicam theologiam esse poeticam de deo.

46 Genealogie deorum gentilium, libro XIV.

47 Il défend ces positions dans le De laboribus Hercolis.

48 Declamatio contra poetices calumniatores.

49 Stella (2010). 
Convivio, des méthodes de lecture réservées à la Bible, sans impliquer explicitement l'idée d'une fonction théologique de la poésie, mais se limitant à adopter des instruments d'interprétation devenus communs. De voir ce choix technique comme le témoignage d'une culture pré-humaniste est un malentendu qui révèle la tentation de forcer les textes du XIV $\mathrm{X}^{\mathrm{e}}$ siècle pour y lire à tout prix des anticipations de l'humanisme.

\section{Albertino Mussato et la conception du poète théologien}

Le pas décisif vers la sacralisation de la poésie sur la base de ses procédés expressifs sera accompli par Albertino Mussato et par Boccace, qui le reprend.

Le cœur de ce tournant ${ }^{50}$, qu'on peut suivre, depuis quelques années, dans l'édition de Chevalier, est la célèbre querelle qui s'alluma en 1315 entre Mussato et le frère dominicain Giovannino da Mantova, maître de grammaire (c'est-à-dire de lettres) au Studium de Padoue. Dans l'épitre IV, Mussato avance sa thèse générale de la sacralité de la poésie, s'appuyant sur deux arguments anciens et et eux aussi destinés à un long succès : celui de l'analogie narrative entre épisodes de la Bible et contes mythologiques, analogie qui attesterait le partage d'une base spirituelle et d'une inspiration divine communes, et celui de la nature poétique de la Bible, ou au moins de quelques-unes de ses parties (comme le Cantique de Moïse et l'Apocalypse). Giovannino lui répond dans un texte qui ne nous est jamais arrivé, mais que l'on peut reconstituer à partir de la réponse de Mussato (ép. 18, dans les éditions modernes). À leurs deux voix, s'ajoutent celle des declarationes qui, dans les manuscrits, accompagnent le texte poétique, et dont Chevalier attribue la rédaction à deux amis de Mussato, appartenant au même milieu pré-humaniste : Castellano di Bassano et Guizzardo di Bologna, commentateurs de l'Ecerinis ${ }^{51}$ dont les arguments Ricklin a démontré tres proches de la lettre à Cangrande. Nous allons analyser les plus significatifs des neuf arguments, et les plus utiles à encadrer le statut de la poésie biblique.

50 Chevalier (2000) XCIII ss. ; les informations ici données sont plus exactes et mises à jour que dans la bibliographie précedente. Voir aussi Greenfield (1981) ; Fitzgerald (2017) chap. 6 Albertino Mussato and Humanist Prophecy, 193-229. Sur l'esthétique de Mussato avant lui Vinay (1949) 113-159 et Dazzi (1964). Le MS. des épîtres poétiques est Venise, Archivio di Stato, ex Brera 277.

51 Les on a récemment involvés pour la coïncidence des expressions de leur commentaire avec des formules de l'Épitre à Cangrande : Chevalier (2000) XCIII. Il s'agit de deux commentateurs de l'Ecerinis, la tragédie avec la quelle Mussato reouvre la tradition du théâtre classique interrompue au Moyen Âge. Le texte de Castellano et Guizzardo a été publié seulement dans un imprimé du 1616, d'où Garin 1965 l'a traduit dans les pages 2-19. Voir Billanovich (1976) 73-74. B. Fiztgerald cite une ed. HR fasc. 7 p. 69 et Cecchini (1985) 95-119. 


\section{Contre-arguments 1 et 2 . La poésie n'est pas un art «divin» car elle ne parle pas de réalités divines}

Giovannino s'oppose à Mussato en rappelant les condamnations que la poésie avait subies de la part de la théologie catholique, depuis Jérôme et Grégoire le Grand, et il reprend le raisonnement de Thomas, qu'on reconnaît de la citation d'Orphée, Musée et Linos $^{52}$, en lui opposant la remarque que les textes poétiques païens, contrairement à la Genèse biblique, ne parlent pas du vrai Dieu, mais de phénomènes célestes (tels Océan et Téthys) ou d'hommes divinisés ${ }^{53}$.

\section{Argument 6. L’emploi biblique de la poésie en confirme la nature sacrée}

Dans Exode 15, Moïse prononce un chant de louanges et d'exultation pour la «victoire» sur les Égyptiens après la traversée de la Mer Rouge. Giovannino admet qu'il s'agit là de poésie ${ }^{54}$, mais, à son avis, ce rapprochement ne justifie pas la poésie en général. Il reconnaît aussi que quelques poètes bibliques exceptionnels, tels qu'Arator et Sedulius, ont versifié la Bible entière ${ }^{55}$, mais cet élément non plus ne permet pas de définir divine la poésie dans son ensemble, tandis que la Bible est entièrement divine. Ce problème, déjà abordé par Aristote à propos d'Empédocle, relève donc de catégories logiques, et non d'un préjugé culturel : «en effet, de même que la science naturelle ou n'importe quelle autre ne saurait être définie Logique, bien qu'elle emploie des procédés de démonstration typiques de la Logique, ainsi toute science qui est susceptible d'être versifiée ne peut pas pour autant être définie "poésie«, bien que la poésie présente ce même mode d'expression » ${ }^{56}$.

52 Voir ci-dessus p. 481-482. L'exemple sera repris par Coluccio Salutati De laboribus Herculis I.9, rr. 25-28 Ullmann: Fuerunt et post eos Museus, Orpheus et Linus, qui nedum carminibus deos colebant, sed ceperunt etiam de diis et ipsorum statu naturaque non medioctriter disputare. Unde et theologi dicti sunt. Chevalier (2000) CV n. 43 renvoie au célèbre passage d'Augustin, Civ. XVIII.14, mais, comme on l'a vu, les références préalables à Salutati sont plus nombreuses et complexes, et peuvent être déchiffrées par l'individuation exacte des textes.

53 C'est un argument de la critique anti-religieuse rationaliste, le soi-disant évémerisme, que la culture chrétienne avait déjà exprimé de façon organique avec Lactance : Institutiones Divinae I.11 et De ira Dei 11.

54 Mussato ep. IV.61-62.

55 Ceci n'est pas du tout exacte: Sedulius versifia l’Évangile, même s’il le fit précéder, dans les premier de ses quatre livres, par une synthése des événements bibliques antérieurs, et Arator se borna aux Actes des apôtres. Le passage de Giovannino, qui est de difficile obtention, dit: Dato etiam quod tota sacra theologia esset metrice tradita, sicut quidam excellentes viri, ut Arator et Sedulius, facere conati sunt, propter hoc tamen poetica non esset dicenda divina.

56 Sicut enim scientia naturalis vel quaelibet alia non potest dici logica, licet utantur modo demonstrandi quem tradidit logicus; sic etiam quaelibet scientia potest tradi metrice, non tamen propter hoc est dicenda poetica, licet talem modum scribendi poetica doceat (Garin 1958, de l'ed. Venezia 1636). 
Le commentaire attribué à Guizzardo et Castellano ${ }^{57}$ interprète cette argumentation en expliquant que, de même que les autres sciences se divisent en théorie et pratique, ainsi la poésie est soit une science théorique, lorsqu'elle compose des histoires à propos d'une vérité, comme Christ le faisait dans les paraboles, soit une science pratique, lorsqu'elle versifie des dictons ou des actions super alia significatione, comme le font Arator et Sedulius. Donc, la poésie serait divine sous son aspect théorique, alors qu'elle serait «technique» (artificialis) du moment où elle est pratique $^{58}$. Il s'agit d'une interprétation spécieuse et aiguë, car, si elle a l'air de décrire l'argument négatif de Giovannino, néanmoins elle l'emploie, en postulant l'équivalence théorique=divine qui en effet n'est pas démontré, pour «sauver » un élément de sacralité de la poésie ; ainsi cherche-t-elle une position de conciliation à laquelle sa source ne visait pas, et elle prouve l'existence d'un milieu intellectuel décidé à soutenir la légitimation de la poésie sans s'opposer à la Scolastique et sans en nier les raisons «scientifiques». L'observation du commentateur pouvait avoir des issues importantes parce qu'il identifie dans la poésie biblique un élément de médiation entre les deux positions : mais ce n'est pas là que se trouve la solution du problème, puisque le contenu de vérité de la poésie ne "transmet» pas sa vérité au code expressif, c'est-à-dire à la poésie. La prémisse implicite est que, de toute façon, lorsqu'elle s'occupe de la Bible, la poésie est divine. Cette prémisse a eu effectivement une grande fortune dans la pratique de la production poétique, mais moins de reconnaissance dans les textes de théorisation poétique que nous connaissons ${ }^{59}$.

\section{Argument 7. L’analogie expressive entre poésie et Bible (symbolisme)}

C'est le nœud de l'entière démonstration : Christ a lui-même communiqué à ses disciples sous forme d'énigmes obscures : nigmata discipulis dixit operta suis (7.38, concernant les paraboles) et les «sacres» poètes suscitent de l'admiration car un sens différent du littéral impose davantage d'attention. Sur cette base, Mussato défend la définition de poésie comme «deuxième philosophie» légitimée par les plus grandes autorités, telles Aristote et Platon, tout en soulignant que même la doctrine juridique, si chère à son interlocuteur, mentionne de temps en temps les vers d’Homère. La réplique de Giovannino s'appuie sur un argument thomiste très solide :

57 Voir ci-dessus, n. 78.

58 Quemadmodum aliae scientiae in practicam et theoricam dividuntur, sic et poetica scientia theorica est, cum quis super veritatem aliquam fabulam fingit, sicut faciebat Dominus noster Iesus Christus in Parabolis. Poetica vero practica est, quum quis quae dicta vel acta sunt super alia significatione in metro describit, ut illi Arator vel Sedulius. Secundum igitur theoricam poetica est divina, secundum autem practicam est ut aliae scientiae artificiales.

59 Par souci de concision, je me réfère à Stella (2001), 42-46 («Legittimità di un'estetica eteronoma»). La position de Mussato sur la poésie dans ses dernières années est étudiée par Fiacchini (2017). 
la ressemblance entre les modes d'expression de la un poésie et ceux des Saintes Écritures n'est qu'apparente. En effet, «la poésie emploie des métaphores pour représenter et plaire, alors que les Écritures divines le font pour voiler le rayon des Écritures mêmes et de la Vérité, qui peut ainsi être cherchée avec plus d'engagement par ceux qui en sont dignes, tout en restant cachée aux indignes. Les Écritures, en effet, sont couvertes pour ne pas s'avilir, et sont dévoilées pour nourrir les âmes ${ }^{60} »$ : la différence en termes de finalité et de substance que l'allégorie vise à souligner, répète, de façon concise, ce qu'exprimait Thomas ${ }^{61}$, et ignore tout à fait la spécificité des paraphrases poétiques de la Bible.

Mussato répond dans la lettre 18 (113 s.), en rappelant à son interlocuteur qu'il avait lui-même cité l'Apocalypse comme étant connotée d'un langage assimilable au langage de la poésie élevée ${ }^{62}$, et il l'invite à lire ce que les novi poete - les poètes bibliques, justement - avaient écrit sur la naissance du Christ ${ }^{63}$, en invoquant quelques vers de celui que, dans l'un des rares versets chrétiens de sa production ${ }^{64}$, il appelle Claudien et qui est, précisément, Claudius Claudianus, que Chevalier confond avec Claudien Mamert. La réplique de Mussato se base donc sur une méprise intentionnelle de l'argument de Giovannino ${ }^{65}$, mais elle saisit la puissance gagnante de la solution anti-classiciste : l'existence d'une poésie capable d'associer la vérité des contenus aux capacités prophétiques et métaphoriques du langage. Ce serait la poésie chrétienne, la poésie biblique, à avoir résolu l'aporie du peu de «contenu de vérité» de la poésie classique. Dans le sillage de Mussato et de ses commentateurs, à la fois Dominici, dans sa querelle contre Boccace-Salutati, et Boccace lui-même développeront cette solution, et ce dernier en tirera une projection de grande envergure, reconstituant un fil commun allant de Juvencus jusqu'à Alain de Lille, à Dante et au Pétrarque-Bucoliques, projection que l'humanisme et l'historiographie moderne ont effacée.

60 Ed. Garin 1958, chap. 10: [dici potest] quod poetica propter hoc videtur habere similitudinem cum dictis Scripturis, quia divina Scriptura metaphoris utitur, sicut poetica, ut patet maxime in Apocalypsi et in libris prophetarum. Sed differunt omnino. Nam poetica utitur metaphoris ad repraesentandum et delectandum, sed Divina Scriptura ad radium divinae Scripturae et veritatis circumvelandum, ut a dignis studiosius inquiratur et indignis occultetur; et ob hoc aperiuntur, ut pascant animam.

61 Vedi ci-dessus, p. $479-482$.

62 Sicut ais vere similatur Apocalis alto / eloquio vatum: restat fatearis ut ipsa / sit de fine boni summi concepta poesis.

63 Et posuere novi simul et cecinere poetae, v. 117.

64 Chevalier dans son commentaire le confonde avec Claudien Mamerte, en citant l'edition de la $P L$ (3, coll. 788-9), tandis qu'il s'agit de Claudien, Carmina minora 32.

65 Giovannino en effet citait la poéticité de l'Apocalipse en tant que partie de l'argumentation de l'interlocuteur; deuxièmement il niait de toute façon que ceci pouvait être un exemple d'analogie entre la Bible et la poésie ancienne, justement parce-que dans les deux typologies de texte l'image métaphorique prend deux rôles et deux poids différents. Á cet argument, l'argument thomiste, Mussato ne répond pas, entrainé par l'enthousiasme qui associait chaque forme d'écriture inspirée dans une légitimation commune. 


\section{Argument 9. Contenus crypto-chrétiens de la poésie.}

Le dernier des neuf arguments élaborés par Giovannino concerne ${ }^{66}$ le contenu chrétien de toute poésie. Mussato rappelait que Virgile avait prédit l'avènement du Christ (dans le célèbre puer de la quatrième des Bucoliques), et que ses vers ont été employés pour exprimer des contenus chrétiens dans les centones de Proba et d'autres auteurs de l'Antiquité tardive. Govannino insiste sur l'antériorité de Virgile qui, le Christ n'étant pas encore né, ne pouvait se dire chrétien, et il considère que la reprise de ses vers dans un contexte altéré est un coup de force, ce que Jérôme avait déjà soutenu. Pourtant Mussato et la declaratio anonyme inversent l'argument en rappelant que les prophètes Isaïe, Ézéchiel, Daniel et les autres parlent eux-aussi du Christ avant qu'il ne soit né, puis le Commentaire loue la capacité d'expression chrétienne de Proba, première auteur de Bibeldichtung en latin, dont Mussato avait accepté la condamnation de la part de Jérôme, mais dont le commentaire cite plusieurs vers pour avérer sa thèse ${ }^{67}$.

\section{Issues de la querelle}

Giovannino conclut en affirmant que les arguments de Mussato sont faibles et obscurs (dubia), et il paraît en prôner le développement d'une façon plus articulée et dans un langage convenable, soit dialectique ${ }^{68}$. En revanche, le commentaire qui clôt le texte explique que la réponse qu'a donnée, à son tour, le poète, une fois bien analysée, dissipe tout doute, mais que, puisque le langage poétique contraint à la synthèse, le lecteur cultivé devra en développer les arguments ${ }^{69}$. Le débat peut apparaître comme un dialogue entre deux approches incompatibles, mais ceci dérive surtout de la différence dans la méthode d'articulation des arguments. Frère Giovannino donne à la discussion une structure professionnelle établie selon les règles dialectique de la Scolastique, tandis que Mussato accumule des intuitions et des références qui ne relèvent que d'un nœud essentiel : l'analogie entre Bible et poésie en termes de procédés expressifs et de contenus sapientiels, une suggestion pleinement médiévale - et non seulement patristique, comme le disent quelques étu-

66 Nostra fides sancto tota est predicta Maroni: / Inclita Centone despice metra Probe.

67 Á ces-ci il ajoint une référence au poéte qui, avec le même esprit, adapta les vers de l'Hercules furens de Sénèque à Christ.

68 Sermo ad hoc conveniens: ici Garin traduit «quando se ne darà l'occasione», tandis que nous croyons que Giovannino se réfère au genre qui convient à la discussion théorique, qui ne peut pas être la poésie mais justement la quaestio.

69 Verum cum sententiae quae metrice insinuantur succinctae sint, sic exigente natura metrorum et poeticae facultatis, si prosaice dissererentur clarius elucescerent, despiciat igitur lector prudens et faciliter per metra correspondentia singulis quaestionibus convenientias discerneret artis poeticae ad veram theologiam, et dubia incunctanter absolvet. 
des -, mais déjà dépassée par la Scolastique. Albertino n'avait pas eu la formation pour pouvoir soutenir ce rapport sous l'aspect technique, mais l'emphatisation enthousiaste de l'approche analogique «dionysienne-eriugénienne» finira par s'imposer pendant l'Humanisme entier, et jusqu'au Romantisme, comme on a démontré dans les colloques sur Bible et poésie à Florence $1998^{70}$ : la poésie, grâce à son langage figuré, transmet des contenus de vérité les cachant sous des images apparemment étrangères, de même que la Bible et la théologie ne parlent de Dieu, entité par définition inexprimable, qu'à travers l'intermédiaire de effacer la virgule figures, allégories, négations, circonlocutions. Par ce retour, apparemment inconscient, au néoplatonisme, Mussato ainsi qu'un certain humanisme contrastent les positions plus «modernes» et «scientifiques» des thomistes, réagissant de façon «conservatrice» aux innovations de ces derniers. L'issue que la Bibeldichtung serait susceptible d'offrir, reste sur l'arrière-fond de la discussion, et n'apparaît que de temps en temps dans les réflexions des différents interlocuteurs, écrasée par le postulat selon lequel, lorsque l'on parle de poésie, l'on entend automatiquement la poésie profane. Sous cet aspect, l'humanisme vainc avant même que ne s'ouvre le conflit, par le simple fait que, à l'exception de quelque personnalités conciliantes tels les auteurs du commentaire, on ne visait pas à trouver un point de rencontre, mais plutôt à affirmer les ambitions d'une nouvelle classe intellectuelle - de genre 'bourgeois', notarial et politique - face à l'ordre établi scolastique et universitaire de genre clérical, et la querelle sur la poésie, qui pendant l'humanisme deviendra débat sur les programmes scolaires, n'est qu'un instrument de cet affrontement de pouvoir social et de la concurrence dans le marché de l'instruction publique.

Pourtant l'hétérogénèse des buts fera de cet instrument polémique l'une des pierres angulaires de la culture européenne de la fin de la Renaissance, puis du Romantisme, et seule une analyse affranchie d'aprioris historiographiques et fermement ancrée aux textes et aux manuscrits, pourra nous en raconter le développement, qui, avant Novalis et Blake ${ }^{71}$, atteindra ses sommets intellectuels dans le dialogue Boccace-Dominici et Salutati-Sanminiato, source directe de la Defence of Poesy di Philip Sidney (1595), des sommets destinés à rester incompréhensibles si on le arrache de l'arrière-plan médiéval que nous avons essayé de résumer.

70 En particulier Pagnini (1999) 39-51; voir aussi, qui naturellement se rattache aux travaux de N. Frye, y compris Frye (1969).

71 La question des rapports entre poésie et disciplines de la connaissance (en particulier la philosophie) se ramifie dans le deux veines platonique et «romantique», déjà dans Francis Bacon (1624, p. 85): Historia ad memoriam refertur, poesis ad phantasiam, philosophia ad rationem. Nietzsche considère la poésie une forme de philosophie, continuation de la pensée mythique. Shelley appelle les poètes, comme Mussato l'avait fait en utilisant l'expression altera philosophia pour la poésie, «philosophes de sublime puissance» (Valentino (2002) 214 n. 87). 


\section{Bibliographie}

\section{A. Sources}

par ordre alphabétique du nom de l'éditeur, sauf pour les gravures anciennes

Epistola fratris lohannini de Mantua ... Mussato poetae paduano invehens contra poeticam, Venetiis 1636.

S. Antonini, Summa Theologica, voll. 4, ex typographia Seminarii: apud Augustinum Carattonium, Veronae 1740.

Bossuat (1953): Alano di Lille, Anticlaudianus, éd. R. Bossuat, Paris, Vrin.

Cecchini (1985): E. Cecchini, Le epistole metriche del Mussato sulla poesia, in Tradizione classica e letteratura umanistica per Alessandro Perosa, a cura di R. Cardini, E. Garin, L. Cesarini Martinelli, G. Pascucci, vol. I, Roma, Bulzoni, 95-119 (con ed. delle lettere I, IV, VII e XVIII).

Chevalier (2000): Albertini Mussati Ecerinis. Epitres metriques sur la poésie, Songe, édition critique, traduction et présentation critique par J.-F. Chevalier, Paris, Les Belles Lettres.

Coulon (1908): Beati Iohannis Dominici Lucula noctis, éd. R. Coulon, Paris, Picard, online in www. archive.org.

Fredborg (1988): The Latin Rethorical Commentaries by Thierry of Chartres, ed. K.M. Fredborg, Pontifical Institute of Mediaeval Studies, Toronto.

Frugoni-Brugnoli (1996): Dante Alighieri, Epistole. Egloge. Questio de aqua et terra, a cura di A. Frugoni, G. Brugnoli et al., Milano, Ricciardi.

Gerson (1966): G. Gerson, Oeuvres complètes, vol. vii/1, L'oeuvre française, Paris Desclée \& C. Gillen (1979): O. Gillen, Herrad von Landsberg, Hortus Deliciarum, Neustadt, Pfalz Verlanganstalt. Glorieux (1953): P. Glorieux, La somme «Quoniam homines» d'Alain de Lille, Archives d'Histoire Doctrinale et Littéraire du Moyen Age 20, 113-364.

Hunt (1961): Iohannis Dominici Lucula noctis, ed. E. Hunt, Notre Dame (Ind.), Notre Dame University Press, 1940, 19602.

Megas (1967): A.Ch. Megas, 'O prooumanistikòs kúklos tes Pádouas (Lovato Lovati-Albertino Mussato) kài oi' tragwdies tou L.A. Seneca, Thessaloniki, 154-161.

Pignoria (1636): Albertini Mussati Historia Augusta Henrici VII Caesaris et alia quae extant opera, edd. L. Pignoria-F. Osio-N. Villani, Venetiis 1636.

\section{B. Études}

Biffi (1999): I. Biffi, La poesia e la grazia nella Commedia di Dante, Milano, Jaca Book.

Biffi (2005): I. Biffi, L'eucaristia in san Tommaso d'Aquino: teologia, mistica e poesia, Siena, Cantagalli 2005.

Billanovich (1976): Guido Billanovich, Storia della cultura veneta.

Cames (1971): Gérard Cames, Allégories et symboles dans l'Hortus deliciarum, Leiden, Brill.

Chenu (1957): Marie Dominique Chenu, La théologie come science au XIIle siècle, Paris (tr. it. La teologia nel XII secolo, Milano, Jaca Book, 1986).

Colnago (2009): Filippo Colnago, Poesia e teologia in Giovanni Scoto l'Eriugena, Roma, Herder Editrice e Libreria

Curtius (1949) = Ernst R. Curtius, «Theologische Poetik im italienischen Trecento», in: Zeitschrift für romanische Philologie 9, 1-15.

Curtius (1992): Ernst R. Curtius, Letteratura europea e medioevo latino, La Nuova Italia, Firenze, La Nuova Italia (ed. or. Francke, Bern 1948). 
Dazzi (1964): Manlio Torquato Dazzi, Il Mussato preumanista (1261-1329): l'ambiente e l'opera, Vicenza, Nei Pozza.

Dronke 1977 = Peter Dronke, «Theologia veluti quaedam poetria: quelqus observations sur la fonction des images poétiques chez Jean Scot», in: Jean Scot Erigène et l'histoire de la philosophie, Paris, Ed. du CNRS, 243-255.

Eco (1975): Umberto Eco, Sviluppo dell'estetica medievale, in Momenti e problemi di storia dell'estetica. 1. Dall'antichità classica al Barocco, Milano, Marzorati.

Eco (1987): Umberto Eco, Arte e bellezza nell'estetica medioevale, Milano, Bompiani.

Facchini (2017): Bianca Facchini, «Albertino Mussato: ultime riflessioni sulla poesia dans »Moribus antiquis sibi me fecere poetam «», in: Albertino Mussato nel VII centenario dell'incoronazione poetica (Padova 1315-2015) cur. R. Modonutti - E. Zucchi, 141-158.

Fitzgerald (2017): Brian Fitzgerald, Inspiration and Authorities in the Middle Ages. Prophets and their critics from Scholasticism to Humanism, Oxford, OUP.

Frye (1969): Northrop Frye, Anatomia della critica, trad. it. Torino.

Garin (1958): Eugenio Garin, Il pensiero pedagogico dell'umanesimo, Firenze, Sansoni.

Gompe (1973): L. Gompe, «Figmenta poetarum», in Literatur und Sprache im Europäischen Mittelalter. Festschrift für Karl Langosch, Darmstadt, Wissenschaftliche Buchgesellschaft, 53-62.

Greenfield (1981): Concetta Carestia Greenfield, Humanist and Scholastic Poetics, 1200-1500, Lewisburg-London-Toronto, Bucknell University Press.

Guthmüller (2013): Bodo Guthmüller, «Giovanni Dominici lettore della »Genealogia«. La polemica religiosa contro lo studio degli antichi poeti in Boccaccio e i suoi lettori. Una lunga ricezione», cur. G.M. Anselmi - G. Baffetti - C. Delcorno - C. S. Nobili, Bologna, Il Mulino, $263-78$.

Jeauneau (1957): Edouard Jeauneau, «L'usage de la notion d'integumentum à travers les gloses de Guillaume de Conches», in: Arch. d'hist. doctr. et litt. du Moyen Âge 32, 35-100.

Joyner (2016): Danielle B. Joyner, Painting the «Hortus deliciarum». Medieval Women, Wisdom, and Time, University Park, PA.

Kristeller (1961): Paul Oskar Kristeller, Renaissance Thought: The Classic, Scholastic, and Humanist Strain, New York, Harper Thorchbooks.

Kristeller (1967): Paul Oskar Kristeller, Le Thomisme et la pensée italienne de la Renaissance, Montréal-Paris, Institut d'études médiévales-Vrin.

Leclercq (1957): Jean Leclercq, L'amour des lettres et le désir de Dieu. Initiation aux auteurs monastiques du Moyen Âge, Paris, Cerf (tr. it. Cultura umanistica e desiderio di Dio: studio sulla letteratura monastica del Medio Evo, Firenze, Sansoni,1965).

Mariétan (1901): Joseph Mariétan, Le problème de la classification des sciences d'Aristote à St. Thomas, Paris, Alcan.

Marrone (1973): S.P. Marrone, «Domenico Silvestri's Defence of Poetry», in: Rinascimento 13, $115-132$.

Meersseman (1958): Giles G. Meersseman, «In libris gentilis non studeant». L'étude des classiques interdite aux clercs au Moyen Âge? «, in: Italia medioevale e umanistica 1, 1-13.

Mersch (2012): Katharina Ulrike Mersch, «Innovationen auf der Grundlage von Traditionen. Kanonikerreform, Selbstreflexivität und Konventsgeschichte im Miniaturenprogramm des Hohenburger »Hortus Deliciarium «», in: Innovation in Klöstern und Orden des Hohen Mittelalters. Aspekte und Pragmatik eines Begriffs cur. Mirko Breitenstein - Stefan Burkhardt - HYPERLINK «http://sip.mirabileweb.it/search-scholars/julia-d\%C3\% BCcker-scholars/1/172295»J. Dücker, Berlin, 225-246.

Mésoniat (1984): Claudio Mésoniat, Poetica Theologia. La Lucula noctis di Giovanni Dominici e le dispute letterarie tra '300 e '400, Roma, Edizioni di Storia e Letteratura. 
Michel (1994): Alain Michel, La Parole et la Beauté. Rhétorique et estéthique dans la tradition occidentale, Paris, Les Belles Lettres.

Minnis (1984): Alastair J. Minnis, Medieval Theories of Authorship: scholastic literary attitudes in the later Middle Ages, London, Scholar Press.

Mixson (2014): James D. Mixson, Giovanni Dominici's «Firefly» Reconsidered in Christianity and Culture in the Middle Ages. Essays to Honor John Van Engen cur. David C. Mengel - Lisa Wolverton, Notre Dame (IN), University of Notre Dame Press.

Nauert (1998): Charles G. Nauert, «Humanism as Method: Roots of Conflict with the Scholastics», in: The Sixteenth Century Journal 29, 427-438.

Nonn (2012): Ulrich Nonn, Mönche, Schreiber und Gelehrte. Bildung und Wissenschaft im Mittelalter, Darmstadt.

Pagani (1995): I. Pagani, La critica letteraria, in Lo spazio letterario del Medioevo, I. Il Medioevo Latino, 3. La circolazione del testo, Salerno Editrice, Roma, 113-162.

Pagnini (1999): M. Pagnini, La Bibbia e i romantici inglesi, in La Scritura infinita. Bibbia e poesia in età romantica e contemporanea (vol. I) a cura di F. Stella, Firenze, Olschki, 39-51.

Pépin (1970): Jean Pépin, Dante et la tradition de l'allégorie, Montréal-Paris, Institut d'études médiévales-Vrin.

Prati (1965): Pino da Prati, Giovanni Dominici e l'Umanesimo, Napoli, Istituto Editoriale del Mezzogiorno.

Roest (2004): Bert Roest, Franciscan Literature of Religious Instruction before the Council of Trent, Leiden.

Romano 2008 = M. Romano, Giovanni Dominici da Firenze: il catalogo delle opere, SISMEL, Firenze 2008.

Ronconi (1976): Giorgio Ronconi, Le origini delle dispute umanistiche sulla poesia (Mussato e Petrarca), Roma, Bulzoni.

Rummel (1995): Erika Rummel, The Humanist-Scholastic Debate in the Renaissance and Reformation, Cambridge (Ma), Harvard University Press.

Siti (1985): Walter Siti, «An American Gnosis. Appunti su critica e religione in margine all'opera di Harold Bloom», in: Rivista di Letteratura Italiana 3, 147-170

Smolak (2000): Kurt Smolak, Die Bibeldichtung als «verfehlte Gattung», in Stella (1999), 14-29.

Stella (1999): Francesco Stella (a cura di), La Scrittura infinita. Bibbia e poesia in età romantica e contemporanea, Firenze, Olschki.

Stella (2001): Francesco Stella, Ad supplementum sensus. Pluralità ermeneutica e incremento di senso nella poetica biblica dal Medioevo a Derrida. Le ragioni di un convegno, in La Scrittura infinita. Poesia e Bibbia in età medievale e umanistica, SISMEL, Firenze, 31-46.

Stella (2001): Francesco Stella, Poesia e teologia. Età patristica e altomedievale, Jaca Book, Milano 2001 (= Stella 2009).

Stella (2009): Francesco Stella, Poesia e teologia. L'Occidente latino tra IV e VIII secolo, in Fondamenti e inizi. IV-IX secolo (Figure del pensiero medievale. Storia della teologia e della filosofia dalla tarda antichità alle soglie dell'umanesimo) dir. I. Biffi e C. Marabelli, Roma-Milano, Città Nuova-Jaca Book, 471-563.

Stella (2010): Francesco Stella, Condanna e difesa della poesia dalla Scolastica all'Umanesimo, paru dans Figure del pensiero medievale VI «La via moderna». XIV e inizi del XV secolo, Milano, Jaca Book.

Treip (1994): Mindele Anne Treip, Allegorical Poetics and the Epic: the Renaissance Tradition to Paradise Lost, Lexington, The University Press of Kentucky.

Valentino (2002): Francesco Valentino, Poesia, fantasia, filosofia. La didattica della creatività nell'esperienza educativa, Armando Editore 2002,

Verdier (1969): Philippe Verdier, «L'iconographie des arts libéraux dans l'art du Moyen Age jusqu'à la fin du quinzième siècle», in: Arts libéraux et philosophie au moyen age. Actes du 
quatrième congrès international de philosophie médiévale. Université de Montréal, Montréal, Canada, 27 août-2 septembre 1967, Montréal-Paris, Institut d'études médiévales-Vrin, $305-355$.

Vinay (1949): Gustavo Vinay, Studi sul Mussato I Il Mussato e l'estetica medievale, Giornale Storico della Letteratura Italiana 126, 113-159.

Wels 2009 = Volkhard Wels, Der Begriff der Dichtung in der frühen Neuzeit, Berlin-Boston, De Gruyter. 
